\title{
Two-magnon scattering in a self-assembled nanoscale network of misfit dislocations
}

\author{
G. Woltersdorf and B. Heinrich \\ Simon Fraser University, 8888 University Drive, Burnaby, British Columbia, Canada V5A 1S6 \\ (Received 15 November 2003; revised manuscript received 5 January 2004; published 26 May 2004)
}

\begin{abstract}
The static and dynamic properties of magnetic $\mathrm{Pd} / \mathrm{Fe}(001)$ ultrathin film crystalline structures prepared on $\mathrm{GaAs}(001)$ templates were investigated by ferromagnetic resonance (FMR) from 10 to $73 \mathrm{GHz}$. It will be shown that the formation of a self-assembled nanoscale network of misfit dislocations in crystalline structures can be detected during the growth by fan-out diffraction features in reflection high electron energy diffraction. This network of defects leads to a strong extrinsic magnetic damping. The out-of-plane measurements of the FMR linewidth have revealed that the extrinsic damping is caused by two-magnon scattering. The contribution to the FMR linewidth from two-magnon scattering is strongly anisotropic and follows the rectangular symmetry of the glide planes of the misfit dislocation network. It will be shown that the observed strong anisotropy in two-magnon scattering can be interpreted by Fourier components of magnetic defects. The angular dependence of the Fourier components results in an effective channelling of scattered spin waves.
\end{abstract}

DOI: $10.1103 /$ PhysRevB.69.184417

PACS number(s): 75.70.-i, 76.50.+g, 81.15.Hi, 61.14.Hg

\section{INTRODUCTION}

The development of magnetic devices operating at high frequencies requires a better understanding of processes responsible for magnetic relaxation. The spin dynamics in the classical limit can be described by the Landau-LifshitzGilbert equation of motion:

$$
\frac{\partial \mathbf{M}}{\partial t}=-\gamma\left[\mathbf{M} \times \mathbf{H}_{\mathrm{eff}}\right]+\alpha\left(\mathbf{M} \times \frac{\partial \mathbf{m}}{\partial t}\right),
$$

where $\mathbf{m}=\mathbf{M} / M_{s}$ is the unit vector in the direction of the magnetic moment $\mathbf{M}, \gamma=g|e| / 2 m c$ is the absolute value of the electron spectroscopic splitting factor, and $\alpha$ is the dimensionless Gilbert damping parameter. The first term on the right-hand side represents the precessional torque in the internal field $\mathbf{H}_{\text {eff }}$ and the second term represents the Gilbert damping torque. ${ }^{1}$ The intrinsic damping in metallic ferromagnets is caused by spin-orbit interaction and it is described phenomenologically by the Gilbert damping torque. ${ }^{2}$ The ferromagnetic resonance (FMR) linewidth [half-width at half maximum (HWHM)] as a function of the microwave frequency $f$ for Gilbert damping is strictly given by

$$
\Delta H(f)=\alpha \frac{2 \pi f}{\gamma} .
$$

A linear frequency dependence of $\Delta H$ is often satisfied in samples with extrinsic damping. ${ }^{1,3}$ However, in this case the extrapolated $\Delta H(0)$ has a nonzero value (zero-frequency offset). The purpose of this paper is to demonstrate that in lattice strained structures the spin dynamics can be strongly affected by a self-assembled network of misfit dislocations. It will be shown that these defects significantly increase magnetic damping by a two-magnon scattering process. The angular dependence of two-magnon scattering exhibits a strong in-plane anisotropy. We will show that FMR is a technique allowing one to determine components of the Fourier transform of magnetic defects which are relevant to magnetic relaxation processes. The observed strong anisotropy in twomagnon scattering originates in the magnetic defects which have the rectangular symmetry given by the glide planes of misfit dislocations. It will be shown that the angular dependence of the $\mathbf{q}$ dependent two-magnon scattering intensity results in channelling of scattered spin waves.

\section{GROWTH, RHEED STUDIES}

Preparation of the ultrathin film structures was carried out by means of molecular-beam epitaxy (MBE). Semiinsulating epi-ready $\operatorname{GaAs}(001)$ wafers (manufactured by American $\mathrm{XTal}$ Technology) were used as templates for the growth of metallic structures. The GaAs substrates were heated to $400^{\circ} \mathrm{C}$ in ultra high vacuum for $10 \mathrm{~h}$ in order to desorb contaminants. Residual oxides and carbon were removed by reactive desorption using an atomic hydrogen source at $400{ }^{\circ} \mathrm{C}$. After $1 \mathrm{~h}$ of hydrogen treatment the surface contamination was below the detection limit of Auger electron spectroscopy. Subsequent annealing up to $600^{\circ} \mathrm{C}$ was monitored using reflection high energy Electron Diffraction (RHEED) until a well ordered $4 \times 6$ reconstruction appeared. The $\mathrm{Fe}$, $\mathrm{Pd}$, and $\mathrm{Au}$ films were grown at room temperature from a thermal source at a base pressure less then $2 \times 10^{-10}$ Torr with a deposition rate of $\sim 1$ monolayer (ML)/min. ${ }^{4}$ The film thickness was monitored by a quartz crystal microbalance and RHEED intensity oscillations, see Fig. 1(a).

GaAs and Fe are well matched in their crystalline structure (half of the unit cell of GaAs is $1.4 \%$ larger than the unit cell of $\mathrm{Fe})$. The $\mathrm{Au}(001)$ surface in-plane lattice spacing is closely matched to the $\mathrm{Fe}(001)$ template $(0.5 \%$ mismatch) when $[110]_{\mathrm{Au}} \|[100]_{\mathrm{Fe}}$. $\operatorname{Pd}(001)$ has a large mismatch (4.4\%) with respect to the $\mathrm{Fe}(001)$ template even when $[110]_{\mathrm{Pd}} \|[100]_{\mathrm{Fe}} \cdot \mathrm{Au}(001)$ grows over Pd with the in-plane cubic axes parallel to those of $\mathrm{Pd}(001)$. The $\mathrm{Pd}$ and $\mathrm{Au}$ lattice spacings are mismatched by $4.9 \%$. The presence of $\mathrm{Pd}$ in crystalline $\mathrm{Au} / \mathrm{Pd} / \mathrm{Fe}(001)$ multilayers provides a large lattice strain which above a critical thickness of Pd is relieved by the formation of misfit dislocations. In this paper we present mainly the results on ultrathin magnetic single-layer films $n \mathrm{Pd} / \mathbf{3 0 F e} / \mathrm{GaAs}(001)$ and $\mathrm{Au} / n \mathrm{Pd} / \mathbf{1 6 F e} / \mathrm{GaAs}(001)$, where the integers represent the number of atomic layers. $n$ was 

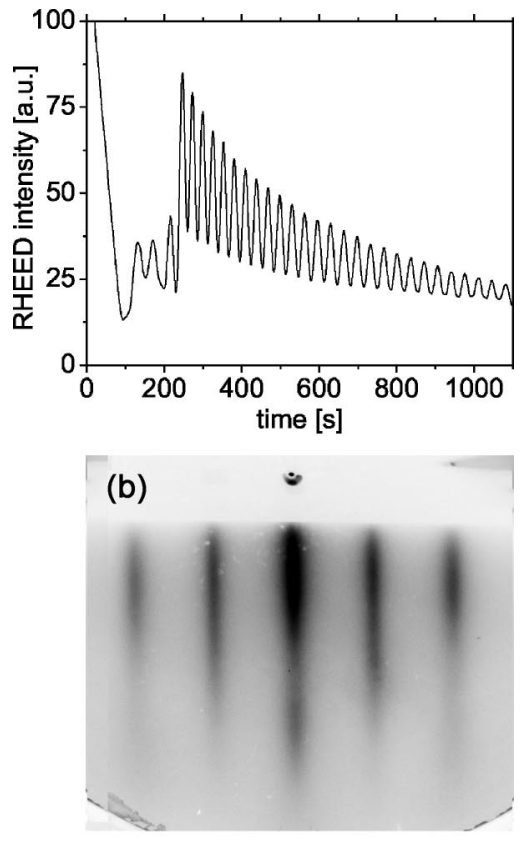

(c)

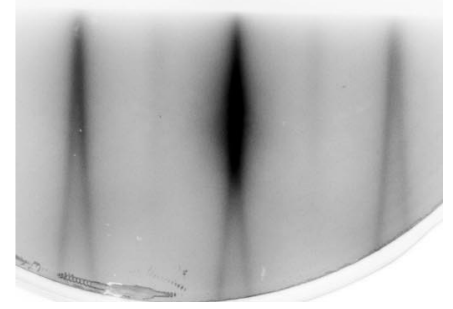

FIG. 1. (a) RHHED intensity oscillations of 30 monolayers (ML) of Fe grown on $\mathrm{GaAs}(001)$. The primary electron beam energy was $10 \mathrm{keV}$. The presence of strong RHEED intensity oscillations after $4 \mathrm{ML}$ indicates a good pseudo-layer-by-layer growth where the surface roughness was confined mostly to two atomic layers. (b) RHEED pattern on $20 \mathrm{Au} / 16 \mathrm{Fe} / \mathrm{GaAs}(001)$ measured with the electron beam along the $[100]_{\mathrm{Au}}$ direction of $\mathrm{Au}(001)$. The real in-plane periodicity is given by the outer streaks. The streaks between the middle streak (zero reciprocal rod) and the outer streaks are caused by the $2 \times 2$ surface reconstruction of surfactant As. (c) shows strong fan out RHEED features on $\mathrm{Au}(001)$ due to a network of misfit dislocations in $20 \mathrm{Au} / 9 \mathrm{Pd} / 16 \mathrm{Fe} / \mathrm{GaAs}(001)$. The electron beam was oriented long $[100]_{\mathrm{Au}}$. Note that the RHEED streak intensities caused by the $2 \times 2$ surface reconstruction are significantly diminished in the presence of misfit dislocations indicating that the long-range order of the surfactant As deteriorated.

between 0 and 300 ML. RHEED intensity oscillations were present during the growth of all layers.

The RHEED pattern of the $\mathrm{Au}(001)$ layer in $\mathrm{Au} /$ $n \mathrm{Pd} / \mathrm{Fe} / \mathrm{GaAs}(001)$ depends on the number of Pd atomic layers, $n$. For $n<4$ the RHEED patterns of Au were the same as those observed in $\mathrm{Au} / \mathrm{Fe} / \mathrm{GaAs}(001)$. They were straight RHEED streaks, see Fig. 1(b), corresponding to a $2 \times 2$ reconstruction of the $\mathrm{Au}(001)$ surface with surfactant As. ${ }^{5}$ For $n \geqslant 4$ the RHEED patterns of $\mathrm{Au}(001)$ developed fan-out streaks when the primary electron beam was oriented along the $\langle 100\rangle_{\mathrm{Au}}$ directions, see Fig. 1(c). For $n \geqslant 9$ the fan-out streaks reached maximum intensity. Fan-out streaks are well known in MBE epitaxy. It was shown by Pukite ${ }^{6}$ that their presence can be related to surface clustering with a rectangular symmetry.

The main diffraction features in RHEED can be described by kinematic scattering. The intensity of diffracted beams is determined by components of the Fourier transform, $S\left(k_{x}, k_{y}\right)$, of surface atoms, ${ }^{7}$ where $k_{x}$ and $k_{y}$ are the inplane $k$ vectors. Pukite's analysis was based on evaluating the surface reciprocal lattice space in the presence of randomly distributed rectangular surface mesas. The mesa cells were generated by an independent geometric distribution of lines along the $x$ and $y$ in-plane coordinates parallel to the atomic steps of mesas. The scattering intensities in this case are given by the product of two Lorentzian functions, ${ }^{6}$

$$
A\left(k_{x}, k_{y}\right)=S^{*} S \sim \frac{\beta}{k_{x}^{2}+\beta^{2}} \frac{\beta}{k_{y}^{2}+\beta^{2}},
$$

where $\beta$ is given by the average size of atomic terraces; $\beta$ $=2 /\langle L\rangle$. The reciprocal surface scattering rods are replaced by reciprocal space sheets oriented along the surface atomic steps. ${ }^{6}$ Their intensities are described by Eq. (3), and the electron diffraction in RHEED is given by the intersection of the Ewald sphere with the reciprocal sheets. This leads to simple diffraction streaks when the electron beam is oriented along the atomic edges (line defects), and results in fan-out streaks for the electron beam oriented $45^{\circ}$ with respect to the atomic steps; see details in Ref. 6. The atomic mesas are one possibility leading to reciprocal sheets. Other systems with rectangular defect distribution can lead to a similar reciprocal space. Parallel dislocation bundles in $\mathrm{Fe}$ on $\mathrm{W}(001)$ also resulted in well-defined reciprocal sheets oriented along $\{100\}_{\mathrm{Fe}}$; see Ref. 8.

Pukite's results allow one to make the following useful conclusion: The presence of RHEED fan-out streaks with $90^{\circ}$ symmetry is due to the formation of a rectangular network of surface line defects. For the RHEED electron beam oriented at $45^{\circ}$ with respect to the direction of line defects the fan-out streak pattern is symmetric and reaches its maximum intensity; while along the line defects (for angles 0 and $90^{\circ}$ ) only simple straight streaks are present.

From the RHEED patterns in Fig. 1(c) one can conclude that the surfaces of the $\mathrm{Au} / n \mathrm{Pd} / \mathrm{Fe} / \mathrm{GaAs}(001)$ structures with $n \geqslant 9$ consist of a network of line defects, which are oriented along the $\langle 110\rangle_{\mathrm{Au}}$ crystallographic directions of $\mathrm{Au}(001)$. J. Woltersdorf investigated the growth of $\mathrm{Pd}$ on a thin foil $\mathrm{Au}(001)$ template using plan view transmission electron microscopy (TEM), ${ }^{9}$ and showed that the strain in $\operatorname{Pd}(001)$ layers thicker than $4 \mathrm{ML}$ is gradually relieved by the formation of dislocation half loops. The glide due to the dislocations creates a rectangular network of surface defect lines oriented along the $\langle 110\rangle_{\mathrm{Au}}$ directions. ${ }^{9,10}$ The lattice mismatches between $\mathrm{Pd}$ and $\mathrm{Au}$ and $\mathrm{Pd}$ and $\mathrm{Fe}$ are similar, and therefore the results of Woltersdorf's TEM studies are relevant to the Pd/ $\mathrm{Fe}(001)$ layers which were employed in our structures. Recent plan view TEM studies by Woltersdorf and Scholz ${ }^{11}$ clearly revealed a network of misfit dislocations, see Fig. 2(a), and reciprocal sheets in the Pd and Au layers ${ }^{11}$ in the 


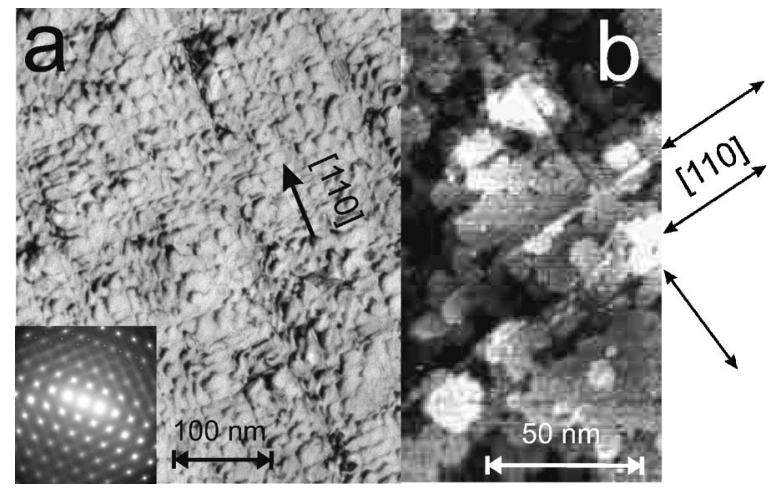

FIG. 2. (a) Plan view TEM image of $90 \mathrm{Au} / 9 \mathrm{Pd} / \mathbf{1 6 F e} /$ $\mathrm{GaAs}(001)$. The arrow indicates the $[110]_{\mathrm{Au}}$ crystallographic direction and the contrast with fourfold symmetry is due to the misfit dislocation network. The inset shows the diffraction pattern where the fourfold symmetry of defects is evident in the reciprocal sheets along the $\langle 110\rangle_{\mathrm{Au}}$ directions. (b) Ex situ STM image of 90Au/9Pd/ $16 \mathrm{Fe} / \mathrm{GaAs}(001)$. The arrows along $\langle 110\rangle_{\mathrm{Au}}$ highlight surface steps which are caused by dislocation glide. The rms roughness was $1 \AA$.

$90 \mathrm{Au} / 9 \mathrm{Pd} / \mathbf{1 6} \mathrm{Fe} / \mathrm{GaAs}(001)$ structure. The lattice strain relaxation in Pd was further confirmed by scanning tunnel microscope (STM) images from the top of $\mathrm{Au}(001)$ layer of the same sample, $90 \mathrm{Au} / 9 \mathrm{Pd} / \mathbf{1 6} \mathbf{F e} / \mathrm{GaAs}(001)$. Figure 2(b) shows surface steps arising from the network of misfit dislocations half loops indicating that the atomic displacements along the $\{111\}_{\mathrm{Au}}$ glide planes propagate through the top Au layer.

\section{FMR STUDIES}

FMR experiments were carried out with the magnetic moment in the film plane (in-plane configuration) and out-ofplane (out-of-plane configuration) using 10, 14, 24, 36, and $73 \mathrm{GHz}$ systems. ${ }^{12}$ The external magnetic field was modu-

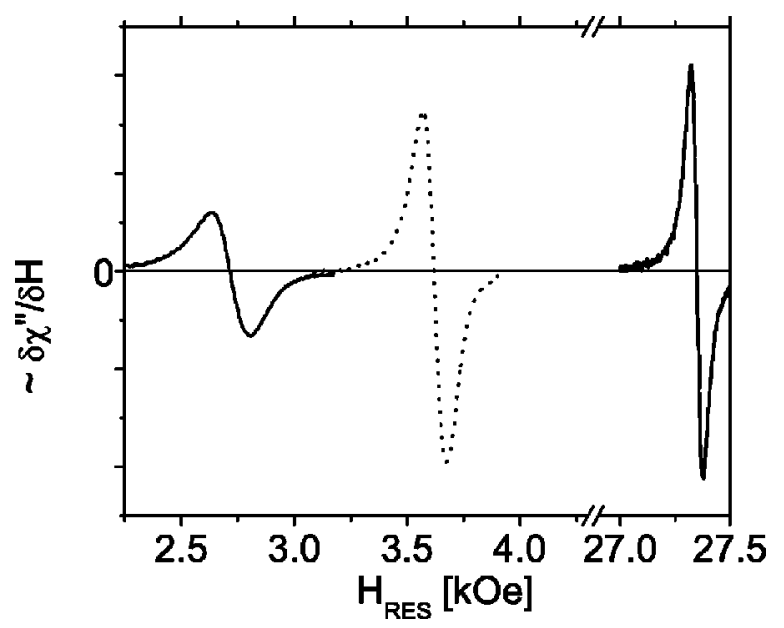

FIG. 3. Typical FMR spectra measured at $24 \mathrm{GHz}$ on the $200 \mathrm{Pd} /$ 30Fe/GaAs(001) sample. The left spectra were taken with the magnetization $\mathbf{M}$ in the plane: $\mathbf{M} \|[100]_{\mathrm{Fe}}$ (solid line) and $\mathbf{M} \|[110]_{\mathrm{Fe}}$ (dotted line). The right spectrum corresponds to the perpendicular configuration $\left(\mathbf{M} \|[001]_{\mathrm{Fe}}\right)$. Note that the FMR linewidths in the in-plane configuration are anisotropic, and the narrowest line is measured in the perpendicular configuration.

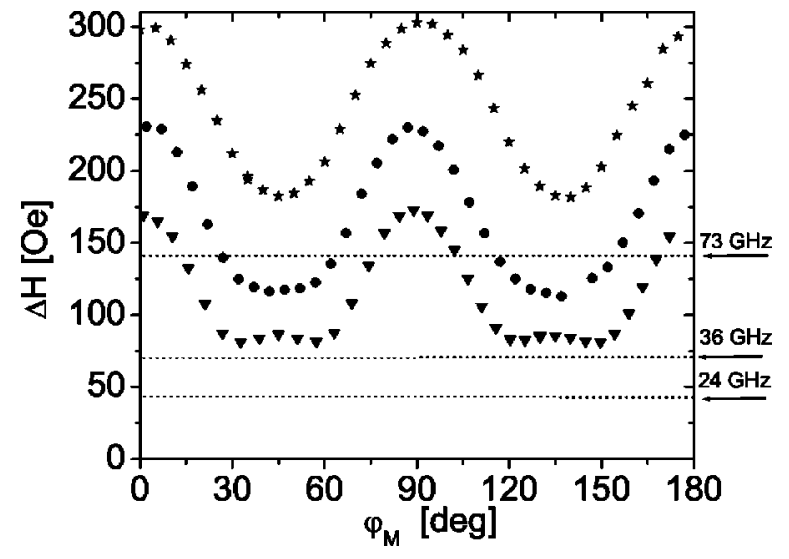

FIG. 4. The ferromagnetic resonance linewidth for the 200Pd/ 30Fe/GaAs(001) film at $73(\star), 36(\bullet)$, and $24(\boldsymbol{\nabla}) \mathrm{GHz}$ as a function of the in-plane angle $\varphi_{M}$ between the magnetization and the $[100]_{\mathrm{Fe}}$ axis. The angles $\varphi_{M}$ were calculated from $\varphi_{H}$ using the magnetic anisotropies and applied field. Dotted lines indicate the linewidth due to intrinsic damping at 73, 36, and $24 \mathrm{GHz}$.

lated with an amplitude of a few oersteds at $80 \mathrm{~Hz}$ and a phase sensitive detection was used to increase the signal-tonoise ratio. ${ }^{12}$ The measured FMR lines were symmetric Lorentzians, see Fig. 3, and their HWHM linewidth was determined using Lorentzian fits.

The in-plane resonance fields and resonance linewidths were measured as a function of the angle $\varphi_{H}$ between the external dc magnetic field $\mathbf{H}$ and the $\mathrm{Fe}$ in-plane $[100]_{\mathrm{Fe}}$ cubic axis.

The magnetic anisotropies of the $30 \mathrm{Fe}(001)$ layer in $n \mathrm{Pd} /$ $\mathbf{3 0 F e} / \mathrm{GaAs}(001)$ and $\mathrm{Au} / n \mathrm{Pd} / \mathbf{1 6 F e} / \mathrm{GaAs}(001)$ are close to those in $\mathrm{Au} / \mathrm{Fe} / \mathrm{GaAs}(001)$ systems of the corresponding $\mathrm{Fe}$ thickness which are not accompanied by a misfit dislocation network. ${ }^{5}$ The easy axes of the $\mathbf{3 0 F e}$ layer are along the $\langle 110\rangle_{\mathrm{Fe}}$ directions due to the uniaxial anisotropy originating at the GaAs interface. ${ }^{5}$ The striking difference between samples with a thick Pd layer in $n \mathrm{Pd} / \mathrm{Fe} / \mathrm{GaAs}(001), n$ $\geqslant 130$, and those with $n \leqslant 110$ was in magnetic damping. The Fe layers in $n \mathrm{Pd} / \mathrm{Fe} / \mathrm{GaAs}(001)$ structures with $n \leqslant 110$ have shown only Gilbert damping which was enhanced by spin pumping of the precessing magnetization of the Fe layer and the spin sink inside the Pd layer. ${ }^{13,5}$ The FMR linewidth in $n \mathrm{Pd} / \mathbf{F e} / \mathrm{GaAs}(001)$ samples with $n \geqslant 130$ was different. In these samples the FMR linewidth was strongly dependent on the angle $\varphi_{M}$ between the magnetization and the crystallographic $\langle 100\rangle_{\mathrm{Fe}}$ axes, showing a distinct fourfold symmetry, see Fig. 4. The minima and maxima in $\Delta H$ are along the $\langle 110\rangle_{\mathrm{Fe}}$ and $\langle 100\rangle_{\mathrm{Fe}}$ crystallographic directions, respectively. It is interesting to note that $\Delta H\left(\varphi_{M}\right)$ has no twofold symmetry. This implies that the chemical bonding between $\mathrm{Fe}$ and the uniaxial dangling bonds of $\mathrm{GaAs}$ at the $\mathrm{Fe} /$ $\mathrm{GaAs}(001)$ interface plays no role in the formation of magnetic defects by the network of misfit dislocations.

The FMR linewidth as a function of microwave frequency also changed in a very pronounced way. The frequency dependence of the FMR linewidth, $\Delta H(f)$, along the $\langle 100\rangle_{\mathrm{Fe}}$ and $\langle 110\rangle_{\mathrm{Fe}}$ directions is shown in Fig. 5. Along the $\langle 110\rangle_{\mathrm{Fe}}$ (easy magnetic axes) and $\langle 1 \overline{1} 0\rangle_{\mathrm{Fe}}$ (hard magnetic axes), the 


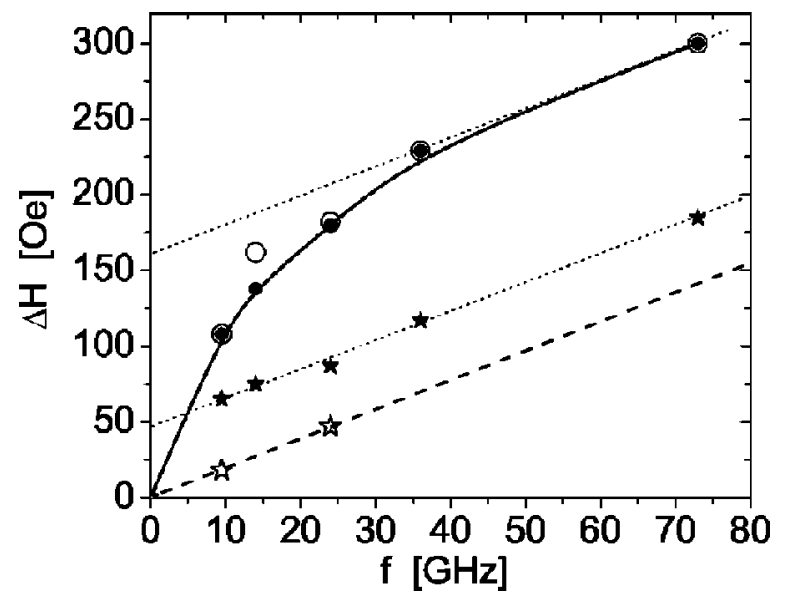

FIG. 5. The frequency dependence of the FMR linewidth for the $200 \mathrm{Pd} / 30 \mathrm{Fe} / \mathrm{GaAs}(001)$ structure along the $\langle 110\rangle_{\mathrm{Fe}}(\star)$ and $\langle 100\rangle_{\mathrm{Fe}}(\bigcirc)$ directions, respectively. $(\bigcirc)$ show $\Delta H$ along $\langle 100\rangle_{\mathrm{Fe}}$ before the dragging contribution to the linewidth was removed (see further details in the text). The purpose of the solid line spline fit is to guide the reader's eye. The dashed line shows the frequency dependence of the intrinsic FMR linewidth (Gilbert damping) obtained by using the $100 \mathrm{Pd} / \mathbf{3 0 F e} / \mathrm{GaAs}(001)$ sample with no magnetic defects in Fe. The spin pumping contribution in $100 \mathrm{Pd} / \mathbf{3 0 F e} /$ $\operatorname{GaAs}(001)$ to $\Delta H$ was already saturated Ref. 14 . is symbols on the dashed line show the FMR linewidth in the perpendicular configuration at 10 and $24 \mathrm{GHz}$ for the $200 \mathrm{Pd} / \mathbf{3 0 F e} / \mathrm{GaAs}(001)$ sample. Note that is are right on the dashed line indicating that in the perpendicular configuration the FMR linewidth $\Delta H$ is only given by the Gilbert damping ( $\alpha=0.006)$ with no zero-frequency offset $\left[\Delta H(0)_{\perp}=0\right]$. The dotted lines indicate the range of microwave frequencies where the slope of $\Delta H(f)$ is close to that expected from the Gilbert damping. Note that the dotted lines have zero-frequency offsets.

FMR linewidth between 10 and $73 \mathrm{GHz}$ was nearly linearly dependent on the microwave frequency, but accompanied by a zero-frequency offset $\Delta H(0)=50$ Oe. However, the slope is close to that corresponding to the intrinsic Gilbert damping obtained in samples without extrinsic damping. The Gilbert damping includes the contribution from spin pumping into a $100 \mathrm{Pd}(001)$ cover layer. ${ }^{14}$ The results are quite different for the FMR measurements with the saturation magnetization along the $\langle 100\rangle_{\mathrm{Fe}}$ directions. One has to realize that for the $30 \mathrm{ML}$ thick Fe film the $\langle 100\rangle_{\mathrm{Fe}}$ directions are neither easy nor hard magnetic axes, and therefore one is not able to avoid dragging of the magnetization behind the applied external field at FMR. The FMR linewidth was determined by first finding the in-plane direction of the external field $\mathrm{H}$ which leads to $\mathbf{M} \|\langle 100\rangle_{\mathrm{Fe}}$ at FMR. The open circles in Fig. 5 show the measured linewidth. However, dragging the magnetization behind the external field results in additional FMR line broadening. This effect is particularly present at low frequencies, because the FMR fields are comparable to the in-plane uniaxial anisotropy field. The dragging is nearly absent at and above $24 \mathrm{GHz}$. In order to remove the dragging contribution the FMR linewidth was evaluated in the following manner: FMR peaks are calculated using the magnetization dragging, and the effective Gilbert damping ( $\left.\alpha_{\text {eff }}\right)$ is adjusted in such a way that the total $\Delta H$ is equal to that observed experimentally (open circles). Then the FMR linewidths without the dragging contribution are obtained by using the effective Gilbert damping $\left[\Delta H=(2 \pi f / \gamma) \alpha_{\text {eff }}\right]$. These are shown in the filled points in Fig. 5. Now one can discuss the data. First, the dependence on the microwave frequency is not described by a simple linear dependence as expected for Gilbert damping. Second, the slope of the FMR linewidth is close to that expected for the intrinsic damping only between 36 and $73 \mathrm{GHz}$, but $\Delta H(0)=160$ Oe is significantly increased compared to the $\langle 110\rangle_{\mathrm{Fe}}$ orientations. Third, below $36 \mathrm{GHz}$ the frequency dependence of $\Delta H$ shows a clear downturn. In fact the frequency dependence for the $\langle 100\rangle_{\mathrm{Fe}}$ orientations resembles the recent calculations by Arias and Mills ${ }^{15}$ of extrinsic damping by two-magnon scattering. A similar frequency dependence of $\Delta H$ was found recently by Twisselmann and McMichael ${ }^{16}$ for Permalloy (Py) films grown on $\mathrm{NiO}$ and Lindner et al. ${ }^{17}$ in $\mathrm{Fe} / \mathrm{V}$ superlattices.

The above increase of the FMR linewidth was found in a large number of samples involving a lattice mismatched layer of Pd. A comparable FMR line broadening was observed in the following Fe layers (highlighted in bold): $90 \mathrm{Au} / n \mathrm{Pd} / \mathbf{1 6} \mathrm{Fe} / \mathrm{GaAs}(001)$ and $20 \mathrm{Au} / \mathbf{F e} / 40 \mathrm{Au} /$ $n \mathrm{Pd} / \mathrm{Fe} / \mathrm{GaAs}(001)$ with $n \geqslant 9$. The samples have one in common. The extrinsic damping is triggered by lattice defects which are caused by a misfit dislocation network. The onset of two-magnon scattering requires a critical thickness of Pd which depends on where the Fe layer is located. For the Fe layer deposited on the Pd spacer the critical thickness for the onset of two-magnon scattering is equivalent to the critical thickness for the formation of misfit dislocations in $\mathrm{Pd}, n \geqslant 4$ found in Pd/Au systems. ${ }^{9}$ In the Fe layer directly grown on $\operatorname{GaAs}(001)$ the formation of magnetic defects requires either a thick Pd layer $(n>130)$ or a combination of a thin $\mathrm{Pd}(n \geqslant 9)$ and a thick Au layer $(>70)$, e.g., $90 \mathrm{Au} / 9 \mathrm{Pd} /$ $16 \mathrm{Fe} / \mathrm{GaAs}(001)$ or $200 \mathrm{Pd} / 30 \mathrm{Fe} / \mathrm{GaAs}(001)$. In all samples the strength of the two-magnon scattering was found nearly independent of the thickness of Fe implying that the twomagnon scattering is a bulk effect in the studied range of $\mathrm{Fe}$ thicknesses (10-40 ML). This behavior is expected in good crystalline films; once the dislocation half loops are generated they propagate along the $\{111\}_{\text {Pd }}$ glide planes and are only stopped either by the film surface ( $\mathrm{Fe}$ grown on $\mathrm{Pd}$ ) or by the $\mathrm{GaAs} / \mathrm{Fe}$ interface (Fe grown on $\mathrm{GaAs}$ ). The extrinsic damping in these magnetic double layers exhibited similar qualitative and quantitative behavior. Since in magnetic bilayers the spin pumping affects the angular dependence of the FMR linewidth due to accidental crossovers of the resonance fields ${ }^{13}$ we limit our presentation to a single magnetic layer in $200 \mathrm{Pd} / 30 \mathrm{Fe} / \mathrm{GaAs}(001)$.

\section{TWO-MAGNON SCATTERING}

Two-magnon scattering provides a simple model for description of extrinsic damping in FMR. In this case inhomogeneous magnetic properties generate scattering of the resonant mode (uniform mode with $q \sim 0$ ) into nonuniform modes ( $\mathbf{q} \neq 0$ magnons). Two-magnon scattering has been extensively used to describe extrinsic damping in 


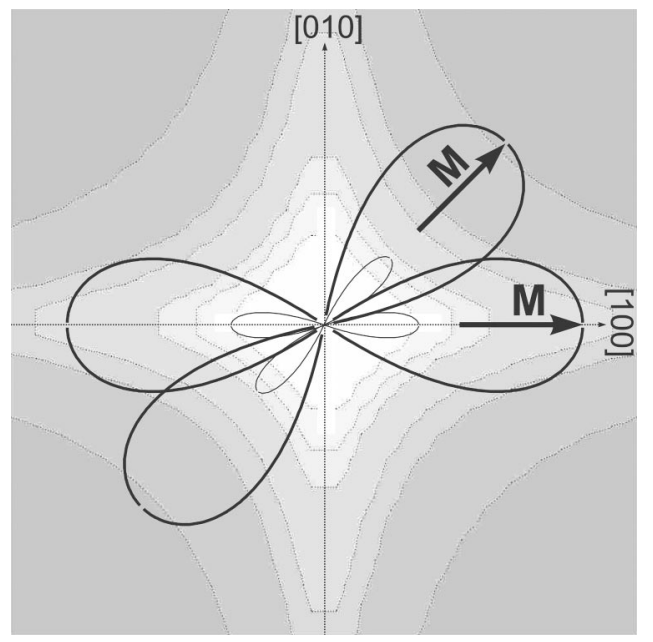

FIG. 6. The black lines show the lobes of degenerate magnons calculated for $73 \mathrm{GHz}$ (big lobes) and $24 \mathrm{GHz}$ (small lobes). The background is a contour plot of the in-plane Fourier components from Eq. (3). The lobes are shown for $\mathbf{M} \|[100]_{\mathrm{Fe}}$ and $\mathbf{M} \|[110]_{\mathrm{Fe}}$. Note that Pukite's type of $I(\mathbf{q})$, which is even scaled to produce anisotropic behavior for small $q$ vectors (large $\langle L\rangle$ ), would result using Eq. (6) only in a weak anisotropy of the FMR linewidth.

ferrites. ${ }^{18-22}$ Patton and co-workers pioneered this concept in metallic films. ${ }^{23}$ In this section we will summarize only those features of two-magnon scattering which are essential for the discussion of our results.

The two-magnon scattering matrix is proportional to components of the Fourier transform of magnetic inhomogeneities $A(\mathbf{q})=\int d \mathbf{r} \Delta U(\mathbf{r}) e^{-i \mathbf{q} \cdot \mathbf{r}}$, where $U(\mathbf{r})$ stands symbolically for a local anisotropy energy. The magnon momentum is not conserved in two-magnon scattering due to the loss of translational invariance, but the energy is conserved. In ultrathin films the magnon $\mathbf{q}$ vectors are confined to the film plane and the magnon energies are given by the dispersion relation which can be found in Ref. 24. For the in-plane orientation the magnon energy with $\mathbf{q}$ parallel to the saturation magnetization decreases its energy with increasing $q$ and eventually due to the effective exchange field crosses the energy of the homogeneous mode at $q=q_{0}$. This means that the magnon with the wave vector $\mathbf{q}_{0}$ is degenerate with the homogeneous mode and can be involved in two-magnon scattering. The value of $q=q_{0}$ decreases with increasing angle $\psi$ between the $\mathbf{q}$ vector and the saturation magnetization. No degenerate modes are available for the angle $\psi$ larger $\operatorname{than}^{24}$

$$
\psi_{\text {max }}=\arcsin \left(\frac{H}{H+4 \pi M_{e f f}}\right)^{1 / 2},
$$

where $H$ is the field at FMR and $4 \pi M_{\text {eff }}$ is the effective demagnetizing field perpendicular to the film surface.

Two-magnon scattering is confined to degenerate magnons following the path of lobes, $\mathbf{q}_{0}(\psi)$, around the direction of the magnetic moment, see Fig. 6.

The two-magnon scattering formally enters the in-plane rf susceptibility $^{24}$ as an additional term $R$ (mass operator) in the denominator,

$$
\chi_{\|}=\frac{M_{s} B}{B_{e f f} H-\left(\frac{\omega}{\gamma}\right)^{2}+i(H+B) \alpha \frac{\omega}{\gamma}+[\operatorname{Re}(R)+i \operatorname{Im}(R)]},
$$

where $B_{e f f}=H+4 \pi M_{e f f}$ is the effective induction, and inplane anisotropies are neglected for simplicity. The real part $\operatorname{Re}(R)$ leads to a shift in the FMR field and the imaginary part $\operatorname{Im}(R)$ provides additional damping. $\operatorname{Re}(R)$ and $\operatorname{Im}(R)$ have to satisfy the symmetry of magnetic inhomogeneities. In a rectangular network of misfit dislocations one expects to get additional two and fourfold anisotropies affecting both the FMR field and magnetic damping. In addition both $\operatorname{Re}(R)$ and $\operatorname{Im}(R)$ are dynamic effects and in general dependent on the microwave frequency. This leads to an important conclusion: Magnetic anisotropies measured by FMR can include frequency-dependent terms which are absent in $d c$ measurements.

The strength of two-magnon scattering in damping as a function of the angle $\varphi_{M}$ of the magnetization with respect to the in-plane crystallographic axis can be tested by evaluating the expression $\operatorname{Im}(R)$. Using the above concept of Fourier components of magnetic inhomogeneities one can write

$$
\begin{aligned}
\operatorname{Im}\left[R\left(\varphi_{M}\right)\right] & \sim \int I(\mathbf{q}) \delta\left(\omega-\omega_{\mathbf{q}}\right) d \mathbf{q}^{3} \\
& =2 \int_{-\psi_{\max }}^{\psi_{\max }} I\left(q_{0}, \varphi, \varphi_{M}\right) \frac{q_{0} d \psi}{\frac{\partial \omega}{\partial q}\left(q_{0}, \psi\right)},
\end{aligned}
$$

where $I(\mathbf{q})$ represents the effective intensity of two-magnon scattering, and $\varphi=\varphi_{M}+\psi$ is the angle of the $\mathbf{q}$ vector with respect to $[100]_{\mathrm{Fe}}$ (defect) axis. The magnon group velocity $\partial \omega / \partial q\left(q_{0}, \psi\right)$ in Eq. (6) is proportional to the strength of the dipolar and exchange fields and represents the dipoleexchange narrowing of local inhomogeneities. ${ }^{18}$ The expression $q_{0} /(\partial \omega / \partial q)\left(q_{0}, \psi\right)$ describes a weighting parameter along the path of two-magnon scattering lobes $\mathbf{q}(\psi)$. It turns out that for a given microwave frequency this factor is nearly independent of $\psi$. This means the whole two-magnon scattering lobe contributes to $\operatorname{Im}(R)$ with an equal weight independent of the angle $\psi$. An exception is the $q$ space close to the origin of the reciprocal space. One has to realize that long-wavelength (small $q$ ) variations in magnetic properties can lead to a simple superposition of local FMR peaks. The extrinsic FMR linewidth in this case merely reflects large length scale sample inhomogeneities and should not be treated by two-magnon scattering. Twisselmann and McMichael $^{16}$ concluded that the FMR linewidth is given by a superposition of local resonances when the characteristic inhomogeneity field is larger than interaction field. ${ }^{25}$ In the range of long-wavelength defects the important part of the interaction field is the magnetostatic contribution $2 \pi M_{s} \mathrm{q} d$ to the magnon energy dispersion, where $d$ is the film thickness. Therefore the FMR spectrum is given by a simple superposition of local FMR peaks when

$$
H_{p} D \geqslant 3 \pi M_{s} d,
$$




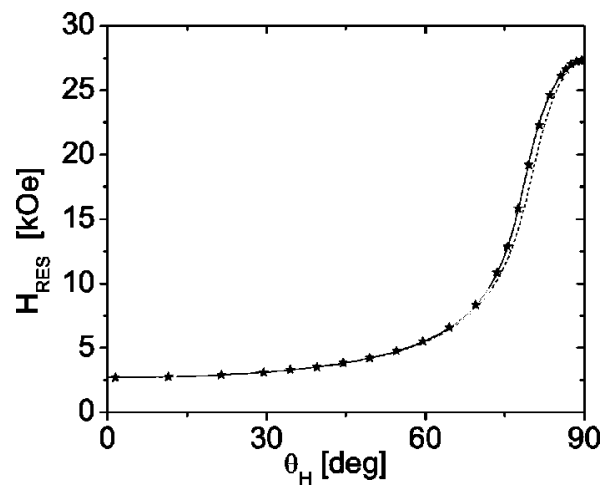

FIG. 7. The ferromagnetic resonance field as a function of the angle $\theta_{H}$ between the sample plane and the applied field $H$ measured at $24 \mathrm{GHz}$. The the magnetic field was rotated in the (001) plane. The parameters required to fit the data are as follows: $4 \pi M_{e f f}=20.6 \mathrm{kG} ; \quad 2 K_{1}^{\|} / M_{s}=330 \mathrm{Oe} ; \quad 2 K_{U}^{\|} / M_{s}=-360 \mathrm{Oe}$; $2 K_{1}^{\perp} / M_{s}=-350$ Oe, where the individual terms describe effective demagnetizing field, fourfold in-plane, uniaxial in-plane, and fourfold perpendicular anisotropy fields, respectively. $g=2.02$ is the $g$ factor accompanying the gyromagnetic ratio $\gamma$. The dashed line shows the fit obtained by fixing $4 \pi M_{e f f}, 2 K_{1}^{\|} / M_{s}, 2 K_{U}^{\|} / M_{s}$, and $g$ factor to the values from the in-plane FMR measurements, see Fig. 10. The only adjustable parameter was $2 K_{1}^{\perp} / M_{s}=-700$ Oe.

where $H_{p}$ is the root-mean-square value of random variations of a local anisotropy field satisfying a Gaussian distribution and $D$ is the corresponding average grain size; see Fig. 4 in Refs. 26. The summation of local FMR signals can result in a genuine zero-frequency offset $\Delta H(0)$; see Ref. 26.

The critical angle $\psi_{\max }$ decreases with an increasing angle $\theta_{M}$ of the magnetization with respect to the sample plane. For $\theta_{M} \geqslant \pi / 4$ no degenerate magnons are available. ${ }^{27}$ The angle $\theta_{H}$ satisfying $\theta_{M} \geqslant \pi / 4$ has to be calculated by minimizing the total magnetic energy. For the 200Pd/30Fe/GaAs sample this angle was $78^{\circ}$ at $24 \mathrm{GHz}$; see Fig. 9. This is an important criterium allowing one to test the applicability of two-magnon scattering in the interpretation of extrinsic damping. $\Delta H$ from the extrinsic damping has to disappear

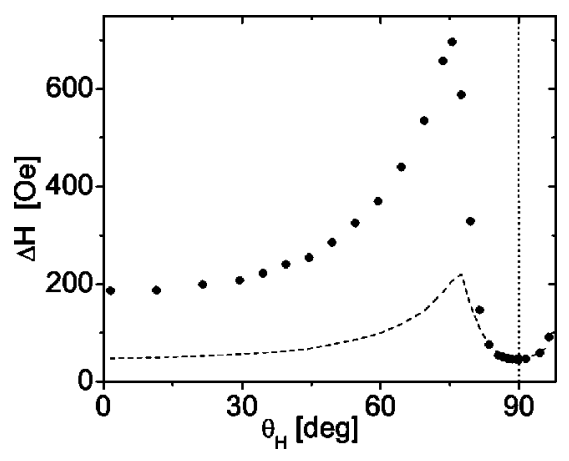

FIG. 8. Measured ferromagnetic resonance linewidth $\Delta H_{m}$ as a function of $\theta_{H}$ at $24 \mathrm{GHz}$. The dots represent the measured data and the dashed line represents the FMR linewidth $\Delta H_{G}(\theta)$ that was calculated using the Gilbert damping from the perpendicular configuration. The peak in the FMR linewidth for $\theta=78^{\circ}$ is caused by dragging the magnetization behind the applied field.

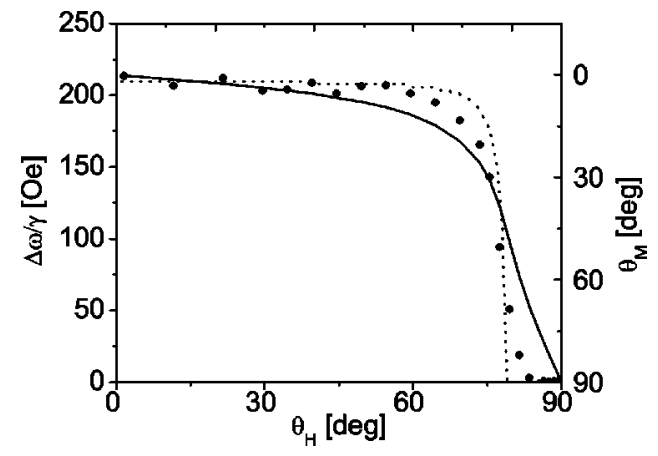

FIG. 9. (O) represent the adjusted frequency FMR linewidth $\Delta \omega / \gamma$ from the extrinsic contribution as a function $\theta_{H}$ at $24 \mathrm{GHz}$. The solid line shows the angle of magnetization $\theta_{M}$ as a function of $\theta_{H}$. The dashed line shows the critical angle $\psi_{\max }$ as a function $\theta_{H}$. Notice that $\psi_{\max }$ describes the angular dependence of $\Delta \omega / \gamma$ quite well. $\psi_{\max }$ was scaled in order to compare it with $\Delta \omega / \gamma$.

when the external field is applied in the vicinity of the film normal. It is interesting to note that the weighing parameter $\mathrm{q}_{0} /(\partial \omega / \partial q)\left(q_{0}, \psi\right)$ is nearly independent of $\theta_{M}$. It somewhat increases very close to the critical angle $\theta_{M}=\pi / 4$ where the two-magnon scattering is switched off.

\section{DISCUSSION OF FMR LINEWIDTH}

The dependence of the FMR field and linewidth on the angle $\theta_{H}$ between the dc magnetic field and the sample plane is shown in Figs. 7 and 8.

The results of measurements of $\Delta H$ as a function of $\theta_{H}$, see Fig. 8, shows that the damping decreases significantly in vicinity of the perpendicular configuration. In fact, the measured $\Delta H$ in the perpendicular configuration at 10 and 24 $\mathrm{GHz}$ was given exactly by the intrinsic damping; see Figs. 3, 5 , and 8 .

The strength of two-magnon scattering as a function of $\theta_{H}$ is usually expressed in terms of the adjusted frequency linewidth $^{22}$

$$
\frac{\Delta \omega}{\gamma}=\left(\frac{d \omega}{d H}\right) \Delta H_{e x t}\left(\theta_{H}\right),
$$

where $d \omega / d H=[\omega(H+\Delta H, \theta+\Delta \theta)-\omega(H, \theta)] / \Delta H$ using the FMR condition for the resonance frequency which includes the in-plane and out-of-plane magnetic anisotropies. It is more convenient to calculate $d \omega / d H$ by picking $\Delta \omega$ and evaluating the corresponding change in $\Delta H$ and $\Delta \theta$ satisfying the resonance condition. The difference between the measured linewidth $\Delta H_{m}\left(\theta_{H}\right)$ and the predicted linewidth $\Delta H_{G}\left(\theta_{H}\right)$ using the intrinsic Gilbert damping determines the contribution arising from the extrinsic damping, $\Delta H_{\text {ext }}\left(\theta_{H}\right)$ $=\Delta H_{m}\left(\theta_{H}\right)-\Delta H_{G}\left(\theta_{H}\right)$.

The dots in Fig. 9 show that the angle $\theta_{M}$ at which the two-magnon contribution rapidly disappears is in excellent agreement with the theoretical prediction of $\pi / 4$. This behavior was found in all samples which were affected by the network of misfit dislocations.

Figure 8 shows convincingly that the FMR linewidth in the vicinity of the perpendicular configuration is given en- 


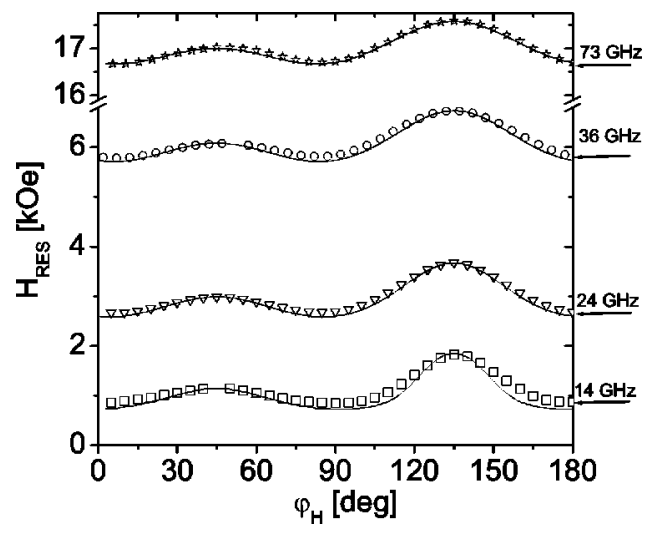

FIG. 10. The ferromagnetic resonance (FMR) fields for the 30Fe layer in $200 \mathrm{Pd} / \mathbf{3 0 F e} / \mathrm{GaAs}(001)$ as a function of the in-plane angle $\varphi_{H}$, where $\varphi_{H}=0$ corresponds to the $[100]_{\mathrm{Fe}}$ direction. Measurements were carried out at $73(\xi), 36(\bigcirc), 24(\nabla)$, and 14 ( $\square$ ) GHz. The solid lines were calculated using the following parameters: The effective demagnetizing field $4 \pi M_{e f f}=18.7 \mathrm{kG}$, inplane uniaxial field $2 K_{u}^{\|} / M_{s}=-360$ Oe, fourfold in-plane anisotropy field $2 K_{1}^{\perp} / M_{s}=330 \mathrm{Oe}$, and the spectroscopic $g$ factor, $g$ $=2.11$. The uniaxial in-plane anisotropy has its hard axis along the $[1 \overline{1} 0]_{\mathrm{Fe}}$ direction. With these parameters the agreement between the measured and calculated FMR fields along $\langle 110\rangle_{\mathrm{Fe}}$ was within 10 Oe for all frequencies. This agreement noticeably deteriorated for the field applied along $\langle 100\rangle_{\mathrm{Fe}}$ where the discrepancy was of the order of 100 Oe. One is not able to get a perfect fit for all microwave frequencies by using one set of parameters indicating that the magnetic anisotropies are partly frequency dependent.

tirely by Gilbert damping. The extrinsic damping as a function of $\theta_{H}$ remains nearly constant until the direction of the magnetic moment is in vicinity of $\pi / 4$ where it abruptly collapses to zero; see Fig. 9.

It follows that the extrinsic damping generated by a selfassembled network of misfit dislocations can be described by the two-magnon scattering mechanism. Since the angular dependence of $\Delta \omega\left(\theta_{H}\right) / \gamma$ is traced well by $\psi_{\text {max }}\left(\theta_{H}\right)$ one can conclude that the two-magnon scattering intensity $I\left(\mathrm{q}, \theta_{H}\right)$ is only weakly dependent on $\theta_{H}$ [see Fig. 9 and Eq. (6)].

The presence of a marked difference in the magnetic parameters which were required for fitting the angular dependence of the FMR field in the parallel and perpendicular configurations, see Figs. 7 and 10, requires a brief explanation. The reason for this discrepancy can be found in Fig. 8. Two-magnon scattering affects the FMR linewidth most strongly when the resonance field increases rapidly with increasing $\theta_{H}$. One can expect that in this range of $\theta_{H}$ the corresponding $\operatorname{Re}(R)$ term is also strongly present. This leads to a noticeable shift in the resonance field compared to that expected using the intrinsic (dc) magnetic anisotropies. The curvature of the FMR field as a function of $\theta_{H}$ in Fig. 7 is very sensitive to the $g$ factor. The change in this curvature due to the contribution of $\operatorname{Re}(R)$ requires to fit the data with a different value of the $g$ factor compared to that corresponding to the intrinsic magnetic properties. An artificially low value $g=2.02$ that was required to fit the curvature in Fig. 7 consequently affected the value of $4 \pi M_{\text {eff }}$ needed to fit the parallel configuration $\left(\theta_{H}=0\right)$. Two-magnon scattering also affected the FMR field in the parallel configuration. Although in this case the contribution of two-magnon scattering to the FMR field is not as strong, and therefore the deviations from a simple fitting [ignoring frequency dependent $\operatorname{Re}(R)]$ are not as vivid. However even in the parallel configuration we were not able to fit the data at all microwave frequencies with the same set of parameters, see Fig. 10. In fact the FMR data in the parallel configuration were fit quite well by assuming a partly frequency dependent in-plane fourfold anisotropy $K_{1}^{\|} \cdot 2 K_{1}^{\|} / M_{s}$ changed from 305 Oe at 14 $\mathrm{GHz}$ to $390 \mathrm{Oe}$ at $73 \mathrm{GHz}$. This is exactly what one should expect, the two-magnon scattering in a rectangular network of misfit dislocations has to satisfy the in-plane fourfold symmetry which leads to a frequency dependent fourfold anisotropy given by the anisotropic contribution of $\operatorname{Re}(R)$.

The two-magnon scattering in samples with a selfassembled network of misfit dislocations showed a very pronounced fourfold in-plane dependence on the angle $\varphi_{M}$ between the saturation magnetization and the crystallographic axis; see Fig. 4. At the same time the functional form of the fourfold anisotropy is dependent on the microwave frequency. These striking features require in depth discussion.

In ultrathin films the Fourier components of magnon scattering are restricted to the in-plane $\mathbf{q}$ vectors and this is common to Pukite's analysis of RHEED diffraction of surface defects. Consequently, one could consider Pukite's in-plane Fourier components for the interpretation of our results. Although one should realize that there are crucial differences. $q_{0}$ in two-magnon scattering is small, just of $\sim 6$ $\times 10^{5} \mathrm{~cm}^{-1}$ at $73 \mathrm{GHz}$ and $\sim 1 \times 10^{5} \mathrm{~cm}^{-1}$ at $14 \mathrm{GHz}$ for a $30 \mathrm{ML}$ thick Fe film. This means that two-magnon scattering is sensitive mostly to the reciprocal space of low $q$ vectors. In RHEED large $\mathbf{k}$ vectors, which are comparable to the reciprocal space of the lattice, are important. However there is even a more profound difference: In magnetism defects can be accompanied by some angular dependent anisotropy. This means that the two-magnon scattering intensity can have an explicit dependence on the direction of the magnetization with respect to the symmetry axis of magnetic defects. This case was recently addressed by Lindner et al. ${ }^{17}$. They observed an anisotropic extrinsic damping [measured along the $\langle 100\rangle$ and $\langle 110\rangle$ axes] for Fe/V superlattices. No detailed dependence of the FMR linewidth as a function of $\varphi_{M}$ was shown. It was assumed that defects were caused by surface steps. By using a simple argument based on the angular dependence of the uniaxial anisotropy they concluded that a rectangular distribution of interface steps results in two-magnon scattering with an anisotropic $\Delta H$ having a $\cos ^{2}\left(2 \varphi_{M}\right)$ dependence. This way the difference in $\Delta H$ along $\langle 100\rangle$ and $\langle 110\rangle$ was explained. This argument can be applied to our system of defects (rectangular network of misfit dislocations). However, this is not the only part which is dependent on the angle with respect to the crystallographic axes. The Fourier components of the scattering intensity have to include two parts. One is explicitly dependent on the angle $\varphi_{M}$ and the other on the wave vector $\mathbf{q}$,

$$
I\left(\mathbf{q}, \varphi_{M}\right)=Q(\mathbf{q}) \cos ^{2}\left(2 \varphi_{M}\right)
$$


In addition one has to consider lower order symmetry terms. In our case this is an isotropic contribution which depends only on the magnitude of $q$. In this case one can write

$$
I\left(\mathbf{q}, \varphi_{M}\right)=Q(\mathbf{q}) \cos ^{2}\left(2 \varphi_{M}\right)+Y(q) .
$$

The $Y(q)$ term corresponds to random distribution of defects. $Q(\mathbf{q})$ has to satisfy the symmetry of defects. The lattice defects in crystalline samples are correlated with crystallographic axes and consequently $Q(\mathbf{q})=Q(q, \varphi)$, where $\varphi$ is the angle between $\mathbf{q}$ and the $[100]_{\mathrm{Fe}}$ defect axis. It turns out that $Q(\mathbf{q})$ is essential for getting the correct angular dependence of $\Delta H\left(\varphi_{M}\right)$ at various microwave frequencies.

$Q(q, \varphi)$ can be expected to be related to the average separation of misfit dislocations. The average separation of misfit dislocations is $\sim 10-20 \mathrm{~nm}$ corresponding to a Fourier component $q \sim 1 \times 10^{6} \mathrm{~cm}^{-1}$. In magnetic scattering one has to consider the exchange coupling within the ferromagnetic film. Lateral inhomogeneities on the scale of $10-20 \mathrm{~nm}$ create large exchange fields that significantly average out the lateral variations of the magnetic anisotropy. This means that the defect length scale of $10-20 \mathrm{~nm}$ does not have to be directly applicable to the discussion of two-magnon scattering.

In order to explain the angular dependence of the twomagnon scattering in our samples one has to address directly the FMR results.

The filled stars in Fig. 5 show $\Delta H(f)$ for $\langle 110\rangle . \Delta H(f)$ has a linear dependence on microwave frequency $f$ with the slope corresponding to Gilbert damping and a modest $\Delta H(0)_{\|} . \Delta H(0)_{\|}$can be caused by long range inhomogeneities (superposition of local FMR lines) and an isotropic term $Y(q)$ in the scattering matrix $I(\mathbf{q})$ [see Eq. (10)]. If one assumes a genuine $\Delta H(0)_{\|}$one has to ask why those longwavelength inhomogeneities were not observed in the perpendicular FMR configuration. Long wave length inhomogeneities of the in-plane uniaxial anisotropy would result in $\Delta H(0)_{\perp}$. It would be $\sim \frac{1}{2} \Delta H(0)_{\|}$and detectable. The absence of $\Delta H(0)_{\perp}$ can be explained by having an inhomogeneous in-plane fourfold anisotropy. It would contribute to $\Delta H(0)_{\|}$but not to $\Delta H(0)_{\perp}$. In the perpendicular FMR $K_{1}^{\|}$ contributes to the free energy with the fourth power in the rf magnetization components and therefore drops out from the resonance condition. One should point out that the perpendicular fourfold anisotropy $K_{1}^{\perp} \cos ^{4}\left(\theta_{M}\right)$ is much stronger, see caption of Fig. 7, and if inhomogeneous would result in $\Delta H(0)_{\perp}$. The absence of $\Delta H(0)_{\perp}$ would suggest that $K_{1}^{\perp}$ is very homogeneous. This is hard to believe considering that $K_{1}^{\perp}$ is mainly caused by defects. In fact it is bigger and has the opposite sign compared to $K_{1}^{\|}$. Inhomogeneity in $K_{1}^{\|}$can also result in an angular dependence of $\Delta H(0)_{\|}$and would follow an eightfold symmetry. It should disappear when the magnetization is oriented half way between $\langle 100\rangle_{\mathrm{Fe}}$ and $\langle 110\rangle_{\mathrm{Fe}}$ where the fourfold anisotropy field is zero. There is some evidence for this behavior in Fig. 4 at $24 \mathrm{GHz}$. However the two additional shallow minima around the $\langle 110\rangle_{\mathrm{Fe}}$ directions indicate that this effect is very weak. This leads to the second possibility which is based on the presence of $Y(q)$. The linear slope of $\Delta H(f)$ was given by the Gilbert

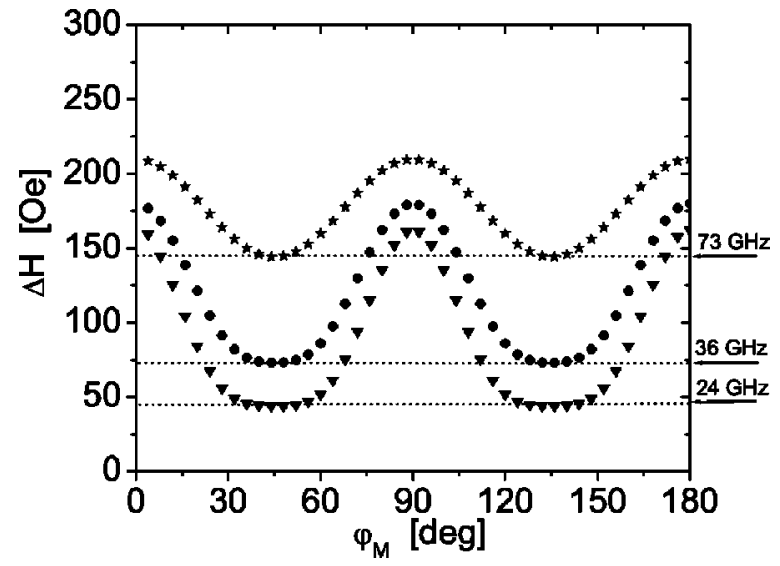

FIG. 11. Calcultated ferromagnetic resonance linewidth at $73(\star), 24(\boldsymbol{\bullet})$, and $14(\boldsymbol{\nabla}) \mathrm{GHz}$ as a function of the in-plane angle $\varphi_{M}$. Where $\varphi_{M}=0$ corresponds to the $[100]_{\mathrm{Fe}}$ direction of Fe. The calculations were carried out using $I\left(\mathbf{q}, \varphi_{M}\right)$ $=Q(\mathbf{q}) \cos ^{2}\left(2 \varphi_{M}\right)$, where $Q(\mathbf{q})$ is given by Eq. (11). Dotted lines indicate the linewidth due to intrinsic damping at 73,36 , and $24 \mathrm{GHz}$.

damping and therefore the two-magnon contribution was constant between 10 and $73 \mathrm{GHz}$. This implies that the twomagnon scattering approaches zero only below $10 \mathrm{GHz}$. A gradual approach to zero in two-magnon scattering below 10 $\mathrm{GHz}$ was observed by Twisselmann and McMichael in Py/ $\mathrm{NiO}$ samples. ${ }^{16}$ The constant two-magnon scattering above $10 \mathrm{GHz}$ in these samples can be accounted for by the $q$ dependence of $Y(\mathrm{q})$.

Our further analysis will be carried out for the angular dependent part of two-magnon scattering. In this paper we tried to determine the main features responsible for the observed two-magnon scattering taking into account the above constraints. Our main emphasis was placed on explaining the in-plane angular dependence of the FMR linewidth and its frequency dependence. A pronounced angular dependence is due to the explicit dependence $\left[\cos ^{2}\left(2 \varphi_{M}\right)\right]$ of the scattering matrix on the angle $\varphi_{M}$. One needs to find $Q(\mathbf{q})$ that accounts for the measured angular dependence $\Delta H\left(\varphi_{M}\right)$ at various microwave frequencies. $Q(\mathbf{q})$ has to satisfy the symmetry of the lattice defects. We used the following ansatz:

$$
Q(q, \varphi)=\cos ^{4}(2 \varphi)
$$

No dependence on $q$ was assumed at this point. The angular dependence of $\Delta H_{2 \mathrm{mag}}$ was evaluated by using the following simple expression:

$$
\Delta H_{2 \mathrm{mag}}=\frac{\operatorname{Im}\left[R\left(\varphi_{M}\right)\right]}{2 H+4 \pi M_{e f f}} .
$$

This equation accounts only partly for the elliptical polarization at FMR for the parallel configuration. No explicit dependence of $\operatorname{Im}(R)$ on the elliptical polarization was considered.

A simple evaluation of $\Delta H_{2 \mathrm{mag}}$ using Eq. (6) explains our experimental results quite well, compare Figs. 4 and 11. The calculations result in a big anisotropy. It is interesting to note that one does not have to rescale the $I\left(\mathbf{q}, \varphi_{M}\right)$ ansatz for each microwave frequency in order to get a reasonable quantita- 


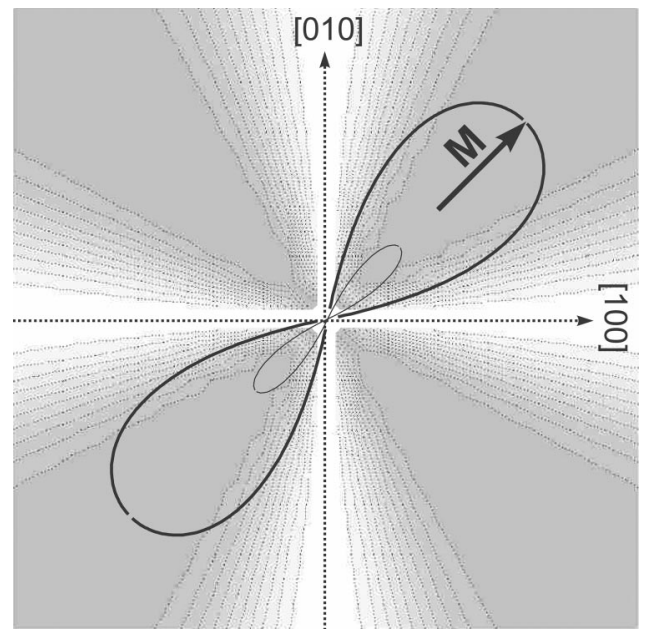

FIG. 12. The two-magnon scattering lobes at 24 and $73 \mathrm{GHz}$ in the $q$ space of the magnetic scattering intensity $Q(q, \varphi)$ given by Eq. (11). The dashed lines are a contour map of Eq. (11). Note that the orientation of lobes (magnetization) affects the angular dependence of FMR linewidth caused by the misfit dislocation network. When the lobes are oriented close to $\langle 110\rangle_{\mathrm{Fe}}$ they have a weaker contribution than those oriented close to $\langle 100\rangle_{\mathrm{Fe}}$.

tive agreement between the calculated and measured $\Delta H\left(\varphi_{M}\right)$. The measured angular dependence of $\Delta H\left(\varphi_{M}\right)$ is sinusoidal at $73 \mathrm{GHz}$. At lower frequencies the curvature around the maxima is bigger than around the minima. This feature is quite pronounced at $24 \mathrm{GHz}$. All these features are well reproduced in the calculations; compare Figs. 4 and 11. The good agreement between the experimental results and calculations for $\Delta H\left(\varphi_{M}\right)$ allows one to make the following observation. The integration along the lobes, see Fig. 12, shows that the scattered magnons are propagating preferentially along the $\langle 100\rangle_{\mathrm{Fe}}$ crystallographic directions. This means that the two-magnon scattering by defects from the network of misfit dislocations leads to channelling of magnons.

In this paper we do not attempt to carry out more detailed comparisons between the two-magnon scattering model and experiment. The relaxation term $R$ would have to be evaluated using Kubo formalism in order to account for the ellipticity of the rf polarization in the in-plane configuration. Such a detailed analysis will allow further refinement of $Q(\mathrm{q}, \varphi)$. These calculations will be carried out in a separate paper where the full theory of two-magnon scattering is employed.

The angular dependent extrinsic damping created by a rectangular network of defects appears to be a common phenomenon. It was observed in our previous studies using the metastable bcc $\mathrm{Ni} / \mathrm{Fe}(001)$ bilayers grown on $\mathrm{Ag}(001)$ substrates, ${ }^{28}$ and $\mathrm{Fe}(001)$ films grown on bcc $\mathrm{Cu}(001) .{ }^{29}$ In the $\mathrm{Ni} / \mathrm{Fe}$ bilayers after depositing $3 \mathrm{ML}$ of Ni the structure went through a major structural change going towards the stable fcc phase of $\mathrm{Ni}(001)$. That resulted in a network of rectangular lattice defects perhaps similar to those observed by Wulfhekel et al. ${ }^{8}$ In this case not only the magnetic damping developed a large anisotropy, but even the in-plane fourfold anisotropy field was enhanced to several kOe which is significantly above that corresponding to cubic bulk Fe ( 0.5 kOe). Coercive fields of several hundred oersteds were reached due to the presence of the enhanced anisotropy and lattice defects and the enhancement was dependent on the $\mathrm{Ni}$ film thickness. ${ }^{30}$ The angular dependence of the FMR linewidth indicated that the defect lines were oriented along the $\langle 100\rangle$ axes of $\mathrm{Fe}(001)$. $\mathrm{Bcc} \mathrm{Cu}(001)$ layers grown on $\mathrm{Ag}(001)$ substrates are another example of such behavior. In this case the bcc $\mathrm{Cu}(001)$ layer went through a lattice transformation after the thickness of the $\mathrm{Cu}$ layer was larger than $10 \mathrm{ML}$. Again a strong anisotropy in $\Delta \mathrm{H}$ was observed for the $\mathrm{Fe}(001)$ films grown on the lattice transformed $\mathrm{Cu}(001)$ substrates. The angular dependence indicated that the defect lines in the $\mathrm{Cu}(001)$ layers and the symmetry axes of magnetic defects in $\mathrm{Fe}(001)$ were along the $\langle 100\rangle$ crystallographic directions of $\mathrm{Fe}(001)$. However, in these samples no significant enhancement of the in-plane fourfold anisotropy was found. Recently we also observed this type of twomagnon scattering in Heusler alloy $\mathrm{NiMnSb}(001)$ semimetal films grown on $\operatorname{InP}(001)$ by the Molenkamp group. ${ }^{31,32}$ In this case $\Delta H$ due to two-magnon scattering surpassed the intrinsic linewidth by a factor of 10 .

\section{CONCLUSIONS}

FMR studies were carried out on lattice strained $\mathrm{Au} / \mathrm{Pd} /$ $\mathbf{F e} / \mathrm{GaAs}(001)$ and $\mathrm{Pd} / \mathrm{Fe} / \mathrm{GaAs}(001)$ structures. It has been shown that the lattice strain in Pd is relieved by a selforganized rectangular network of misfit dislocations which have an average separation of $\sim 10-20 \mathrm{~nm}$. The network of misfit dislocations is revealed by fan-out RHEED streak patterns in the $\mathrm{Au}(001)$ layers. The lattice defects driven by the dislocation network resulted in strong two-magnon scattering. The observed strong anisotropy of the two-magnon scattering with respect to the in-plane orientation of the magnetization originates in uniaxial magnetic anisotropies associated with glide planes of the misfit dislocations. Consequently the fourfold rectangular network of misfit dislocations results in two magnon scattering proportional to $\cos ^{2} 2 \varphi_{M}$. The two-magnon scattering was found independent of the Fe film thickness. This implies that the glide due to the misfit dislocation network propagates right through the whole multilayer structure and associated magnetic defects represent bulk properties. RHEED fan-out streaks and twomagnon scattering have shown a strong in-plane angular dependence. Their angular dependence satisfies the symmetry of the lattice defects. The angular dependence of twomagnon scattering was discussed by using the intensities of the Fourier transform of magnetic defects. We were able to demonstrate that the measurements of the FMR linewidth as a function of the angle of the saturation magnetization with respect to the crystallographic axes and microwave frequency allow one to determine the main features of the magnetic scattering intensities in the range of small $\mathbf{q}$ vectors. The required angular dependence of the $\mathbf{q}$ dependent scattering intensity, $Q(\mathbf{q})$, results in channelling of scattered spin waves. Two-magnon scattering also leads to additional anisotropies which are dependent on the microwave frequency. The magnetic anisotropies created by the two- 
magnon scattering in a self-assembled network of misfit dislocations resulted (using constant magnetic anisotropies in the interpretation of the out-of-plane FMR measurements) in an unrealistic $g$ factor and effective demagnetizing field $4 \pi M_{\text {eff }}$.

Our extensive FMR studies have shown that the presence of angular dependent damping and frequency dependent anisotropies is in general a "smoking gun" indicating lattice defects. In all our studies we have not found any in-plane angular dependent damping that would be of intrinsic origin. In fact, our interpretation of the anisotropic FMR linewidth in $20 \mathrm{Au} / 30 \mathrm{Fe} / 40 \mathrm{Au} / 4 \mathrm{Pd} /[\mathrm{Pd} / \mathrm{Fe}]_{5} / 16 \mathrm{Fe} / \mathrm{GaAs}(001)$ and $\mathrm{Au} /$ $30 \mathrm{Fe} / 40 \mathrm{Au} / 9 \mathrm{Pd} / 16 \mathrm{Fe} / \mathrm{GaAs}(001)$ structures by an anisotropic Stoner enhancement factor in spin pumping ${ }^{33-35}$ was incorrect. In both structures the anisotropic part of the FMR linewidth was caused by the two-magnon scattering mechanism described above.

\section{ACKNOWLEDGMENTS}

The authors thank K. Myrtle for his assistance during the course of this work. We thank Professor J. Woltersdorf and Dr. R. Scholz for providing a plan view TEM image of the misfit dislocation network [Fig. 2(a)]. We would like to acknowledge the valuable discussions with Professor D. L. Mills and Professor C. E. Patton. Financial support from the National Research and Engineering Council of Canada (NSERC) and the Canadian Institute for Advanced Research (CIAR) is gratefully acknowledged. G.W. acknowledges the German Academic Exchange Service (DAAD) for financial support. B.H. acknowledges the Alexander von Humboldt Foundation and Professor J. Kirschner for generous support provided during his stay at the Max Planck Institute in Halle where this manuscript was partly written.
${ }^{1}$ B. Heinrich and J.F. Cochran, Adv. Phys. 42, 523 (1993).

${ }^{2}$ B. Heinrich, R. Urban, and G. Woltersdorf, J. Appl. Phys. 91, 7523 (2002).

${ }^{3}$ R. Urban, B. Heinrich, G. Woltersdorf, K. Ajdari, K. Myrtle, J. Cochran, and E. Rozenberg, Phys. Rev. B 65, 020402 (2002).

${ }^{4}$ T.L. Monchesky, B. Heinrich, R. Urban, K. Myrtle, M. Klaua, and J. Kirschner, Phys. Rev. B 60, 10242 (1999).

${ }^{5}$ R. Urban, G. Woltersdorf, and B. Heinrich, Phys. Rev. Lett. 87, 217204 (2001).

${ }^{6}$ P.R. Pukite, S. Batra, and P. Cohen, Proc. SPIE 796, 22 (1987).

${ }^{7}$ A. Arrott, Magnetic Ultrathin Film Structures I, edited by J. A. C. Bland and B. Heinrich (Springer-Verlag, Berlin, 1994).

${ }^{8}$ W. Wulfhekel, F. Zavaliche, F. Porrati, H. Oepen, and J. Kirschner, Europhys. Lett. 49, 651 (2000).

${ }^{9}$ J. Woltersdorf, Appl. Surf. Sci. 11/12, 495 (1982).

${ }^{10}$ J. Woltersdorf and E. Pippel, Thin Solid Films 116, 77 (1984).

${ }^{11}$ G. Woltersdorf, B. Heinrich, J. Woltersdorf, and R. Scholz, J. Appl. Phys. (to be published).

${ }^{12}$ B. Heinrich and J. A. C. Bland, Ultrathin Magnetic Structures II (Spinger-Verlag, Berlin, 1994), Sec. 3.1, pp. 195-222.

${ }^{13}$ B. Heinrich, Y. Tserkovnyak, G. Woltersdorf, A. Brataas, R. Urban, and G. Bauer, Phys. Rev. Lett. 90, 187601 (2003).

${ }^{14}$ J. Foros, G. Woltersdorf, B. Heinrich, and A. Brataas (unpublished).

${ }^{15}$ R. Arias and D.L. Mills, J. Appl. Phys. 87, 5455 (2000).

${ }^{16}$ D. Twisselmann and R. McMichael, J. Appl. Phys. 93, 6903 (2003).

${ }^{17}$ J. Lindner, L. Lenz, K. Kosubek, K. Baberschke, D. Spoddig, R. Meckenstock, J. Pelzl, Z. Frait, and D. Mills, Phys. Rev. B 68, 060102(R) (2003).

${ }^{18}$ M. Sparks, Ferromagnetic Relaxation Theory (McGraw-Hill, New York, 1966).
${ }^{19}$ E. Schlömann, Phys. Chem. Solids 6, 242 (1958).

${ }^{20}$ R. LeCraw, E.G. Spencer, and C.S. Porter, Phys. Rev. 110, 1311 (1958).

${ }^{21}$ S. Geschwind and A.M. Clogston, Phys. Rev. 108, 49 (1957).

${ }^{22}$ M.J. Hurben, D.R. Franklin, and C.E. Patton, J. Appl. Phys. 81, 7458 (1997).

${ }^{23}$ C.E. Patton, C.H. Wilts, and F.B. Humphrey, J. Appl. Phys. 38, 1358 (1967).

${ }^{24}$ R. Arias and D.L. Mills, Phys. Rev. B 60, 7395 (1999).

${ }^{25} \mathrm{R}$. McMichael (private communication).

${ }^{26}$ R. McMichael, D. Twisselmann, and A. Kunz, Phys. Rev. Lett. 90, 227601 (2003).

${ }^{27}$ D. Mills and S. Rezende, in Spin Dynamics in Confined Magnetic Structures II, edited by B. Hillebrands and K. Ounadjela (Springer-Verlag, Berlin, 2003), pp. 27-57.

${ }^{28}$ B. Heinrich, S. Purcell, J. Dutcher, K. Urquhart, J. Cochran, and A. Arrott, Phys. Rev. 64, 5334 (1988).

${ }^{29}$ Z. Celinski and B. Heinrich, J. Appl. Phys. 70, 5935 (1991).

${ }^{30}$ B. Heinrich, Z. Celinski, H. Konno, A. Arrott, R. Ruhrig, and A. Hubert, Mater. Res. Soc. Symp. Proc. 313, 485 (1993).

${ }^{31}$ B. Heinrich, G. Woltersdorf, R. Urban, A. Mosendz, G. Schmidt, P. Bach, L.W. Molenkamp, and E. Rozenberg, J. Appl. Phys. (to be published).

${ }^{32}$ A. Koveshnikov, G. Woltersdorf, J.Q. Liu, B. Kardaz, A. Mosendz, B. Heinrich, K.L. Kavanagh, P. Bach, A.S. Bader, C. Reuster, C. Gould, G. Schmidt, L.W. Molenkamp, and C. Kumpf, J. Appl. Phys. (to be published).

${ }^{33}$ B. Heinrich, G. Woltersdorf, R. Urban, and E. Šimanek, J. Magn. Magn. Mater. 258, 376 (2003).

${ }^{34}$ B. Heinrich, G. Woltersdorf, R. Urban, and E. Šimanek, J. Appl. Phys. 93, 7545 (2003).

${ }^{35}$ G. Woltersdorf, R. Urban, and B. Heinrich, Mater. Res. Soc. Symp. Proc. 746, 67 (2003). 